\title{
A Dirty Paper Coding Scheme for the Multiple Input Multiple Output Broadcast Channel
}

\author{
Balakrishna Saradka, Srikrishna Bhashyam, Andrew Thangaraj \\ Department of Electrical Engineering \\ Indian Institute of Technology Madras \\ Chennai 600036 \\ Email:\{ee09s007, skrishna, andrew\}@ee.iitm.ac.in
}

\begin{abstract}
Dirty paper coding (DPC) is known to achieve the capacity region of a Gaussian Multiple Input Multiple OutputBroadcast channel (MIMO-BC). Practical DPC schemes using finite length codes are still being actively studied. In this paper, we design a zero-forcing DPC (ZF-DPC) scheme using trellis shaping and Low Density Parity Check (LDPC) codes for a MIMO-BC with two transmit antennas and two users (receivers), each with one antenna. This is an extension of an earlier design for the single antenna Gaussian broadcast channel. One of the important aspects of the DPC code design is the introduction of a one block delay that enables the channel encoder (and decoder) and the shaping encoder (and decoder) to operate independently. In the ZF-DPC method, the MIMO precoder ensures that one user has no interference. The other user uses DPC to combat interference. The performance of this method is compared using simulations with the capacity limit and simpler precoder based methods like Minimum Mean Square Error-Vector Perturbation (MMSE-VP) and zero-forcing beamforming (ZF-BF).
\end{abstract}

\section{INTRODUCTION}

In the cellular downlink, base stations and mobiles use multiple antennas in order to increase spectral efficiency. This downlink channel is a non-degraded Multiple Input Multiple Output Broadcast (MIMO-BC) channel. The sum capacity for the non-degraded Gaussian-MIMO broadcast channel was derived in [1] and shown to be achieved using Dirty Paper Coding (DPC). Later, in [2], it was shown that the DPC rate region coincides with the capacity region. Practical code designs for DPC have been studied in [3]-[7]. In the designs in [3]-[6], joint shaping and coding encoders (or iterative decoders) are required at the transmitter (or receiver). In [7], for the single antenna Gaussian broadcast channel, joint encoding and iterative decoding between the shaping and channel codes are avoided by introducing a one-codeword delay at the transmitter and the shaping of symbols from current message bits combined with parity bits from the previous codeword. In this work, we design codes for the MIMO-BC using this idea from [7].

Other simpler sub-optimal linear and non-linear precoding schemes have also been proposed for the MIMO-BC. The simplest form of linear precoding is Zero-Forcing BeamForming $(\mathrm{ZF}-\mathrm{BF})$. In $\mathrm{ZF}-\mathrm{BF}$, the precoder is simply the pseudo-inverse of the channel matrix. However, this method suffers when there is a large spread of the singular values of the channel matrix. Regularized inversion of the channel matrix and vector perturbation (a non-linear precoding method) can help to address this problem [8], [9].

In this paper, we design a zero-forcing DPC (ZF-DPC) scheme using trellis shaping and Low Density Parity Check (LDPC) codes for a MIMO-BC with two transmit antennas and two users each with one antenna. This is an extension of the design for the single antenna Gaussian broadcast channel in [7]. BER performance of the proposed DPC scheme is compared with the capacity limit and Minimum Mean Square Error-Vector Perturbation (MMSE-VP) and ZF-BF techniques. Since the shaping and channel coding encoders and decoders do not have to be joint, the complexity of the proposed scheme is moderate compared to other DPC designs.

\section{MODEL}

We consider the downlink scenario with the transmitter having 2 antennas and 2 receiving users, each with single antenna. There is no cooperation channel among the users. A block diagram of such a system is shown in Fig. 1. This type of channel can be represented by well known equation

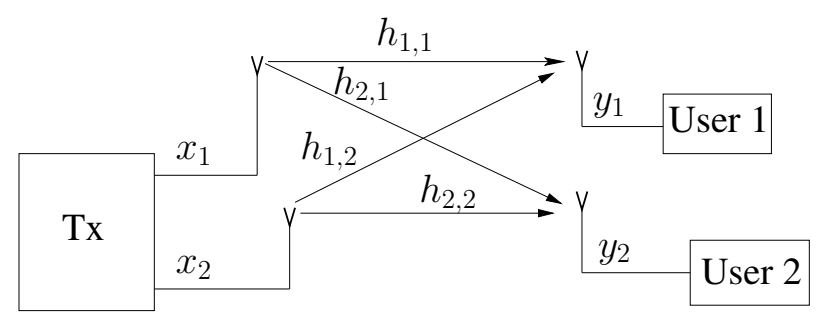

Fig. 1. MIMO-BC model

$\mathbf{y}=\mathbf{H} \mathbf{x}+\mathbf{w}$, where $\mathbf{x}=\left[\begin{array}{ll}x_{1} & x_{2}\end{array}\right]^{T}$ is the transmitted vector and $\mathbf{w}=\left[\begin{array}{ll}w_{1} & w_{2}\end{array}\right]^{T}$ is the complex Additive White Gaussian Noise (AWGN) vector with mean zero and covariance $\mathbf{I}(2 \times$ 2) identity matrix. The channel matrix $\mathbf{H}_{2 \times 2}$ with elements $h_{i, j}$ is fixed and this perfect channel state information (CSI) is assumed to be known completely to the transmitter as well as to both users. Each user's information bits are shaped and channel-coded, and the symbols are chosen from a M-QAM constellation to get symbol vector $\mathbf{d}$. The transmit vector $\mathbf{x}$ is obtained from precoding the symbol vector $\mathbf{d}$ by a MIMO precoder matrix $\mathbf{P}$ such that $\mathbf{x}=\mathbf{P d}$. The transmitter has average power constraint of $P_{t}$ units so that $E\left\{\|\mathbf{x}\|^{2}\right\}<P_{t}$. 
The received vector $\mathbf{y}=\left[\begin{array}{ll}y_{1} & y_{2}\end{array}\right]^{T}$ for the 2 users can be written as:

$$
\begin{aligned}
& y_{1}=h_{11} x_{1}+h_{12} x_{2}+w_{1} \\
& y_{2}=h_{21} x_{1}+h_{22} x_{2}+w_{2}
\end{aligned}
$$

\section{CAPACITY}

In [1], Caire and Shamai derived the sum capacity (total throughput) for the above channel to be:

$R=\left\{\begin{array}{l}\log \left(1+\left|\mathbf{h}_{1}\right|^{2} P_{t}\right), P_{t} \geq P_{1} \\ \log \frac{P_{t} \operatorname{det}\left(\mathbf{H} \mathbf{H}^{\mathbf{H}}+\operatorname{tr}\left(\mathbf{H} \mathbf{H}^{\mathbf{H}}\right)^{2}-4\left|\mathbf{h}_{2} \mathbf{h}_{1}^{H}\right|^{2}\right.}{4 \operatorname{det}\left(\mathbf{H} \mathbf{H}^{\mathbf{H}}\right)}, P_{t} \leq P_{1}\end{array}\right.$

where $\mathbf{h}_{1}, \mathbf{h}_{2}$ are the rows of channel matrix $\mathbf{H}, P_{1}=$ $\frac{\left|\mathbf{h}_{1}\right|^{2}-\left|\mathbf{h}_{2}\right|^{2}}{\operatorname{det}\left(\mathbf{H H}^{\mathrm{H}}\right)}$ and it is assumed that $\left\|\mathbf{h}_{1}\right\| \geq\left\|\mathbf{h}_{2}\right\|$. The sum-rate can be obtained using above equation for any given channel $\mathbf{H}$. When rate for each user is fixed the minimum power required to achieve this can be obtained from the capacity region in [2]. Fig. 2 is a plot of the sum rate as a function of transmitted power for two cases: (a) sum capacity as in [1], (b) maximum sum rate when the two users are required to transmit the same rate. In Fig. 2, we choose

$$
\mathbf{H}=\left[\begin{array}{cc}
0.7071 e^{2.37 j} & 0.8660 e^{2.14 j} \\
e^{2.37 j} & 0.5 e^{0.87 j}
\end{array}\right]
$$

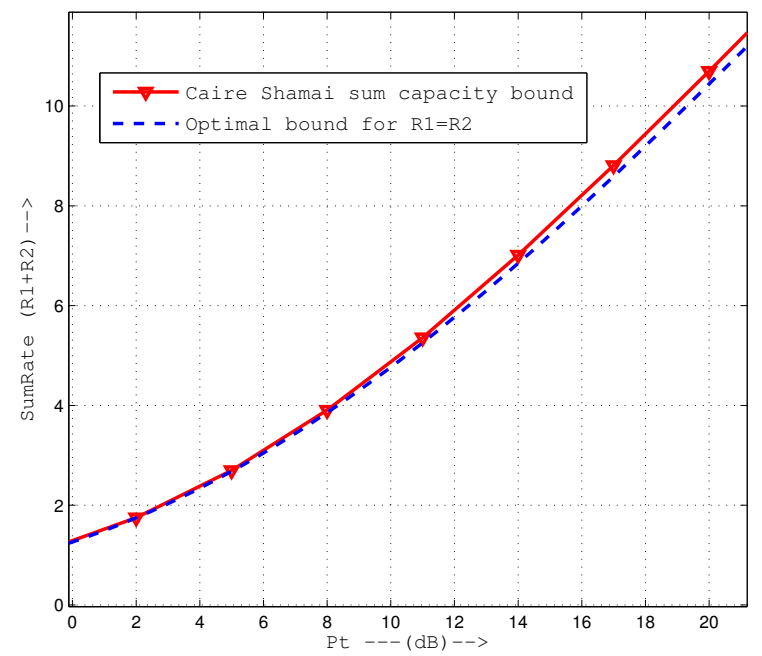

Fig. 2. Capacity bounds $2 \times 2$ MIMO-BC for the given channel $\mathbf{H}$

\section{ZF-DPC WITh TRELlis ShaPING AND LDPC CODE}

\section{MIMO Precoding}

The zero-forcing DPC (ZF-DPC) method proposed in [1] for MIMO-BC is used. The channel matrix $\mathbf{H}$ can be decomposed using Gram-Schmidt orthogonalization as $\mathbf{H}=\mathbf{G Q}$, where $\mathbf{G}$ is a lower triangular matrix and $\mathbf{Q}$ is an orthonormal matrix such that $\mathbf{Q Q}^{H}=\mathbf{I}$ ( $\mathbf{I}$ is a $2 \times 2$ identity matrix). The transmitter precodes the vector $\mathbf{u}$ to get $\mathbf{x}=\mathbf{Q}^{H} \mathbf{R u}$ (See Fig.
3), where the power allocation matrix, $\mathbf{R}=\left[\begin{array}{cc}r_{11} & 0 \\ 0 & r_{22}\end{array}\right]$. Suppose $\mathbf{G}=\left[\begin{array}{cc}g_{11} & 0 \\ g_{21} & g_{22}\end{array}\right]$, we get the received vector to be $\mathbf{y}=\mathbf{G R u}+\mathbf{w}$, which simplifies to:

$$
\begin{aligned}
& y_{1}=g_{11} r_{11} u_{1}+w_{1} \\
& y_{2}=g_{22} r_{22} u_{2}+g_{21} r_{11} u_{1}+w_{2}
\end{aligned}
$$

Thus, we get one interference-free channel and another channel with interference. Here, $u_{1}$ carries the message for user 1 , and $u_{2}$ carries the message for user 2 . User 1 sees no ,interference. User 2 sees an interference term $g_{21} r_{11} u_{1}$. In the case of a MIMO broadcast channel, both the messages are known at the transmitter non-causally. Therefore, the effect of interference at user 2 can be removed using DPC for the message of user 2, i.e., $u_{2}$ is the DPC-coded transmission for user 2. Power allocation matrix entries are computed as in [5].

\section{DPC Scheme}

Dirty paper coding [10] is a technique of canceling known interference at the transmitter. For a channel $\mathbf{Y}=\mathbf{X}+\mathbf{S}+$ $\mathbf{W}$, where $\mathbf{X}=\left[X_{1}, X_{2} \ldots X_{L}\right]$ is the sequence of powerlimited transmitted symbols with power $P_{x}=\frac{E\left[\|\mathbf{X}\|^{2}\right]}{L}$, $\mathbf{S}=\left[S_{1}, S_{2} \ldots S_{L}\right]$ is a known interference sequence at the transmitter and $\mathbf{W}$ is colored or white Gaussian noise vector with $P w=\frac{E\left[\|\mathbf{W}\|^{2}\right]}{L}$, the capacity is the same as if we have no interference $\mathbf{S}$, i.e, $\mathbf{Y}=\mathbf{X}+\mathbf{W}$. A lattice based DPC strategy was proposed in [4] where the transmitter sends $[\mathbf{v}-\alpha \mathbf{S}]$ $\bmod M$ with elements of $\mathbf{v}$ restricted to the symbol set of the elements of $\mathbf{X}$ and $\alpha=P_{x} /\left(P_{w}+P_{x}\right)$ is an MMSE scaling factor [10]. The receiver decodes using $\hat{Y}=(\alpha Y) \bmod M$.

The interference at user 2 is known at transmitter and can be canceled using DPC by selecting the value $\beta=-\alpha \frac{g_{21} r_{11}}{g_{22} r_{22}}$, where $\alpha$ is the MMSE factor.

The rest of the DPC scheme shown in Fig. 3 is based on the design in [7]. The lattice based DPC scheme proposed for degraded broadcast channel [7] is extended to the $2 \times 2$ MIMO$\mathrm{BC}$ case. A lattice-based method that uses a combination of a convolutional code for sign-bit shaping and an LDPC code for channel coding is used. The details are explained in the rest of this section.

\section{Combining Shaping and Channel Coding}

Sign-bit shaping [11] is a method of constellation shaping that modifies the sign of transmitted symbols using redundant bits to choose cosets of convolutional codes. The redundant bits are chosen for the whole block of bits using a trellis of a convolutional decoder so as to optimize a given constraint. The power of the transmitted symbols is usually taken as the minimization constraint. In the interference-free case, this results in choosing constellation points with Gaussian-like distribution. In DPC, the minimization of the power implements the latticequantization after subtraction of interference.

The block diagram of the proposed scheme is shown in Fig. 3. The encoder works with a block of $L$ symbols at a given time. The $i^{t h}$ user has $J$ information bits that are split as $P$ 


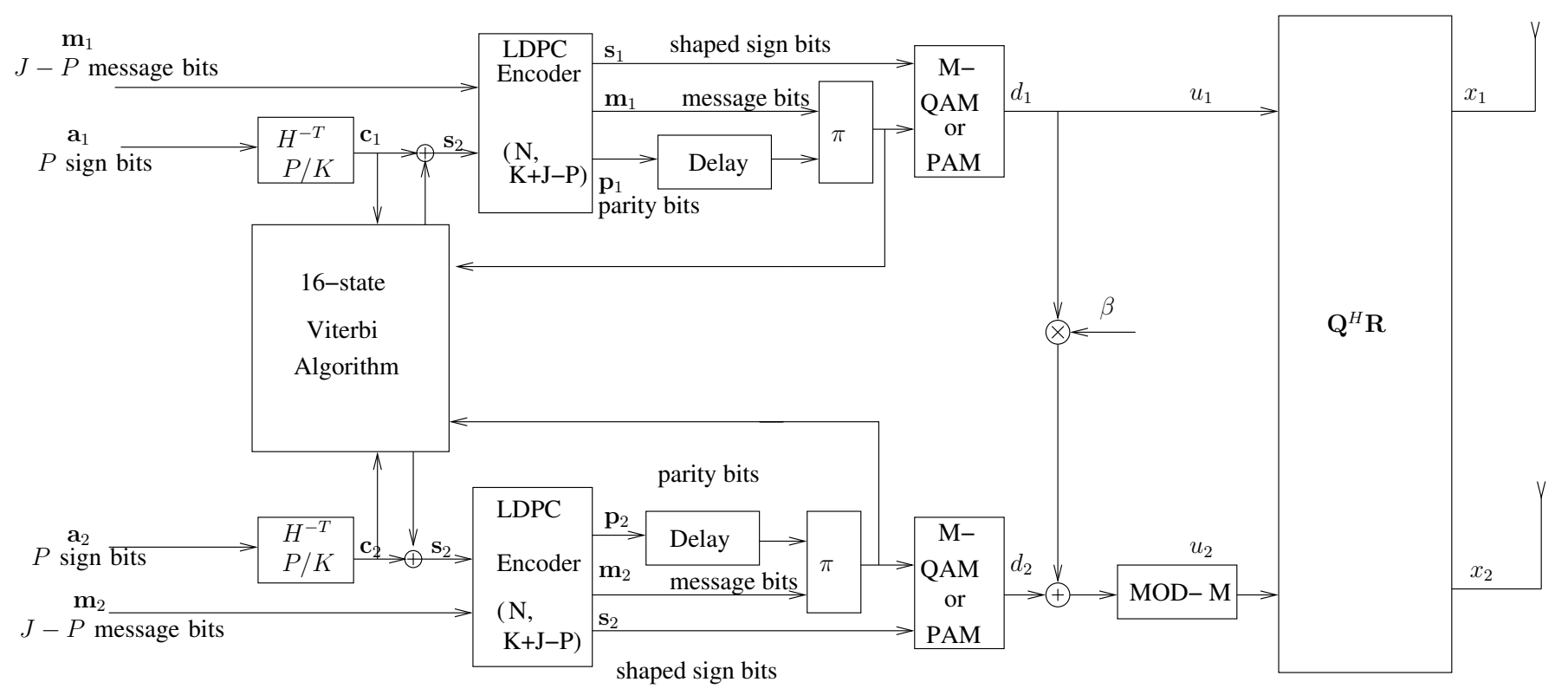

Fig. 3. MIMO Trellis Shaping DPC for $2 \times 2$

sign bits $\mathbf{a}_{i}^{T}=\left[\begin{array}{llll}a_{1}^{i} & a_{2}^{i} & \cdots & a_{P}^{i}\end{array}\right]$ and $J-P$ message bits $\mathbf{m}_{i}^{T}=\left[\begin{array}{llll}m_{1}^{i} & m_{2}^{i} & \cdots & m_{J-P}^{i}\end{array}\right]$. A rate $1 / 2$ non-systematic convolutional code with 4 states is used for sign-bit shaping. The sign bits are passed through the rate- $1 / 2$ inverse-syndrome former having polynomial $H^{-T}=[D, 1+D]$ to map to a coset leader. The output of inverse-syndrome former $\mathbf{c}_{i}^{T}=$ $\left[\begin{array}{llll}c_{1}^{i} & c_{2}^{i} & \cdots & c_{K}^{i}\end{array}\right]$ is the sequence of bits taken as initial signbit sequence. A joint 16-state Viterbi Algorithm (VA) decoder for the convolutional code $C_{U}$ with generator matrix

$$
G_{U}=\left[\begin{array}{cc}
{\left[1+D^{2}, 1+D+D^{2}\right]} & {[0,0]} \\
{[0,0]} & {\left[1+D^{2}, 1+D+D^{2}\right]}
\end{array}\right]
$$

determines the final shaped-bit sequence $\mathbf{s}_{i}^{T}=$ $\left[\begin{array}{llll}s_{1}^{i} & s_{2}^{i} & \cdots & s_{K}^{i}\end{array}\right]$ for each user jointly. The branch metric for Viterbi algorithm is chosen to minimize of the sum of the transmitted power in the two antennas. A $(N, K+J-P)$ LDPC encoder with rate $(K+J-P) / N$ computes parity bits $\mathbf{p}_{i}^{T}=\left[\begin{array}{llll}p_{1}^{i} & p_{2}^{i} & \cdots & p_{N}^{i}\end{array}\right]$ from the sign-bits and message-bits. In order to combine shaping and channel coding, the parity bits are been delayed by one block of $l$ symbols. This means that the parity bits of the previous block are used in the current block to determine the sign bits and the modulated symbol. The delayed parity bits and message bits are interleaved in an interleaver $\pi$ before being modulated to M-QAM symbols. The number of symbols in a given block $L=\frac{N}{\log _{2} M}$ is assumed as an integer. Careful selection of the bits-to symbol mapping is done such that the shaped-bits determines the sign of the constellation symbols $d_{i}$ for each user. The bits to symbol mapping is chosen to be the same for both dimensions of the M-QAM constellation. The mapping $[000,001,010,011,100,101,110,111] \longrightarrow$ $A[1 / 2,3 / 2,7 / 2,5 / 2,-7 / 2,-5 / 2,-1 / 2,-3 / 2]$ in each axis is used for our 64-QAM simulation. This bits-to-symbol mapping is chosen because: (i) flipping of the sign-bit provides a significant change in the transmitted energy of the symbols and (ii) bits are almost as gray coded so that Bit Interleaved Coded Modulation (BICM) for LDPC coding is feasible.

\section{Decoder}

The decoder for this scheme is similar for all users except the scaling factor and is shown in Fig. 4. Bits are decoded by computing $\hat{Y}_{i}=\left(\alpha Y_{i}\right) \bmod M$. The receiver $\bmod$ operation can be replaced by searching $\hat{Y}$ in a M-QAM constellation repeated multiple times in both dimensions [3]. The repeated constellation in each dimension $A_{R}=\{A-$ $r M, \cdots, A-M, A, A+M, \cdots, A+r M\}$ where the $A$ is the transmitter constellation and $r$ is taken to be a sufficiently large value. The receiver computes LLR for each bit as

$$
L_{i}=\frac{\sum_{s \in A_{R}, b i t i=0} \exp \left(\frac{-\left(\hat{Y}_{j}-s\right)^{2}}{2 \beta}\right)}{\sum_{s \in A_{R}, b i t i=1} \exp \left(\frac{-\left(\hat{Y}_{j}-s\right)^{2}}{2 \beta}\right)} .
$$

The LLRs for the sign bits and message bits of block $T$ are used with the LLRs of the parity bits in block $T+1$ for LDPC decoding. Thus, the one block delay of parity bits at the transmitter is compensated before decoding. The inverse permutation for parity bits and message bits are also applied before the LDPC decoder decodes the message and shaped bits. The sign bits are recovered using the syndrome former $H^{T}$ from the shaped bits to get original sign bits.

\section{Simulation Results}

We simulate the BER (averaged over the two users) performance of the proposed ZF-DPC scheme for a sum rate of $4 \mathrm{bits} / \mathrm{s} / \mathrm{Hz}$ (each user with equal rate) and plot it in Fig. 


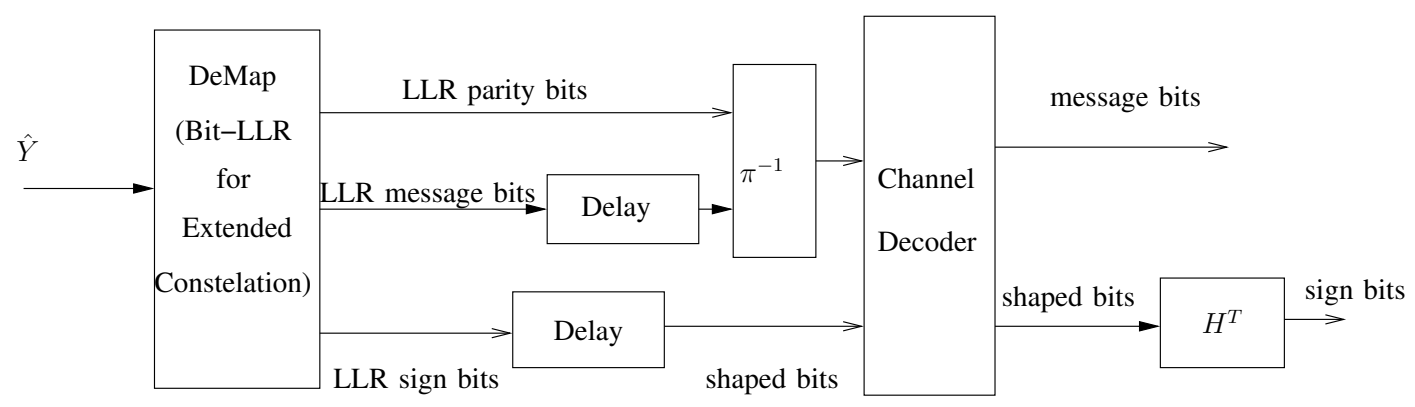

Fig. 4. User module

5. The channel matrix $\mathbf{H}=\left[\begin{array}{cc}0.7071 e^{2.37 j} & 0.8660 e^{2.14 j} \\ e^{2.37 j} & 0.5 e^{0.87 j}\end{array}\right]$ is taken as in [5] for comparison purposes. A shaping code with code rate $1 / 2(=P / K)$ is used for shaping. An irregular LDPC code $(30000,15000)$ with code rate 0.5 having bitnode degree distribution of $0.4691 x+0.3171 x^{2}+0.0103 x^{5}+$ $0.05085 x^{6}+0.1096 x^{8}+0.04305 x^{2} 9$ (edge perspective) and check-node distribution $0.0489 x^{7}+0.9511 x^{8}$ is used as the channel code. These degree distributions are optimized for binary transmission over AWGN channels. Further optimization for the M-QAM constellation is a possible extension of this work. The parameters as shown in the block diagram Fig. 3 are $P=5000, J=10000, K=10000$ and $M=64$ for our simulations. A simple row-column interleaver of row size 174 is used for interleaving parity and message bits before QAM modulation. The MMSE factor, $\alpha=0.71$ is selected by simulation. The overall rate for each individual user is 2 bits/channel use.

We also simulated a ZF-DPC design where we employing our ZF-DPC scheme for the 2 quadrature channels (I and Q) individually using 8-PAM in each dimension, thereby achieving the same overall rate. The performance of this scheme was slightly worse than the 64-QAM design. Note that in the 64-QAM design the sign bits for both the dimensions are jointly optimized

We compare our method with the following: (i) TCQ/TTCM ZF-DPC method proposed in [5], (ii) sub-optimal precoding methods such as MMSE-VP and Zero Forcing Beam Forming (ZF-BF) [9], and (iii) the capacity limit. The result for TCQ/TTCM ZF-DPC is taken from the simulation result in [5]. In case of MMSE-VP, a sphere encoder is used to select the transmitted vector from an extended constellation. We choose an LDPC code $(30000,10000)$ of rate-1/3 with bit node distribution $0.239268 x+0.109947 x^{2}+0.345878 x^{3}+$ $0.304904 x^{1} 4$ (edge perspective) and check node degree distribution $0.454772 x^{4}+0.390687 x^{5}+0.154541 x^{7}$ to code the bits for the individual user rate of $2 \mathrm{bit} / \mathrm{sec} / \mathrm{Hz}$ in 64-QAM constellation. In case of $\mathrm{ZF}-\mathrm{BF}$, the precoding matrix is taken as $\mathbf{H}^{H}\left(\mathbf{H H}^{H}\right)^{-1} \mathbf{R}$. The ZF-DPC method is observed to be $1.8 \mathrm{~dB}$ better than MMSE-VP and $2.4 \mathrm{~dB}$ better than ZF$\mathrm{BF}$ at around $3 \times 10^{-4}$ BER. Based on the capacity limit, the minimum transmitter power $P_{t}$ required for a sum rate of $4 \mathrm{bit} / \mathrm{sec} / \mathrm{Hz}$ is found to be $8.3 \mathrm{~dB}$. From Fig. 2 and our simulation performs $4.3 \mathrm{~dB}$ away from the capacity limit. This method performs $1.9 \mathrm{~dB}$ poorer than the best known coding method for MIMO-BC called TTCM/TCQ method with ZFDPC in [5]. The major loss components are the loss due to LDPC coding, shaping loss and modulo loss. Also, note that our method does not require joint shaping and coding as in [5] using a joint trellis. Further improvement in our scheme is possible by optimization of the LDPC code for 64-QAM and the bit mapping used in our design. At present, the coding loss of the LDPC code used seems to be the major component of the overall loss.

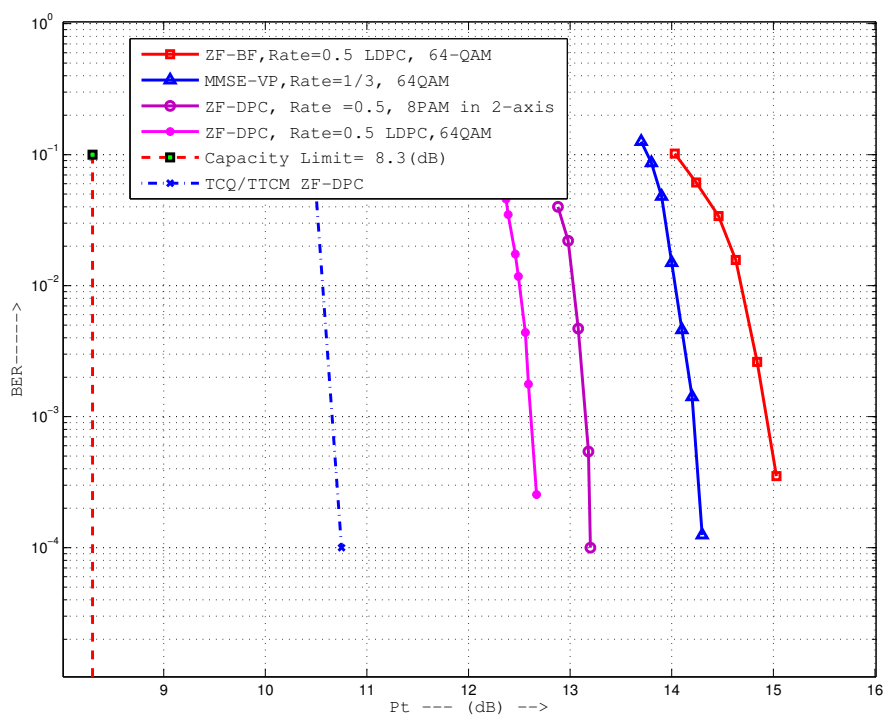

Fig. 5. Comparison results of the scheme for $2 \times 2$ MIMO-BC for sum-rate of $4 \mathrm{bits} / \mathrm{sec} / \mathrm{Hz}$

\section{CONCLUSION}

We designed a ZF-DPC scheme for a non-degraded $2 \times 2$ MIMO-BC channel and studied its BER performance using simulations. The main advantage of our scheme is that we have integrated a shaping technique as well as an LDPC code with the introduction of a simple block delay component for the parity bits at the encoder and a ZF-DPC. This removes the requirement for joint shaping and channel coding. We also compare our method with MMSE-VP and ZF-BF methods and show the gains. The gap from the capacity has also 
been calculated. Reducing this gap with further LDPC code optimization is currently under study.

Other future directions are: (1) Optimal DPC as in [5] instead of ZF-DPC using our design with LDPC codes and sign-bit shaping, (2) Optimizing the LDPC degree distribution specifically for the chosen M-QAM modulation and bit-tosymbol mapping, (3) Designs for higher rates.

\section{ACKNOWLEDGMENT}

The authors would like to thank Shilpa Gadiraju for valuable discussions.

\section{REFERENCES}

[1] G. Caire and S. Shamai, "On the achievable throughput of a multiantenna gaussian broadcast channel," IEEE Transactions on Information Theory, vol. 49, no. 7, pp. 1691 - 1706, July 2003.

[2] H. Weingarten, Y. Steinberg, and S. Shamai, "The capacity region of the gaussian multiple-input multiple-output broadcast channel,' IEEE Transactions on Information Theory, vol. 52, no. 9, pp. 3936 -3964, Sept. 2006.

[3] S. ten Brink and U. Erez, "A close-to-capacity dirty paper coding scheme," in International Symposium on Information Theory, June-July 2004, p. 533.

[4] U. Erez, S. Shamai, and R. Zamir, "Capacity and lattice strategies for canceling known interference," IEEE Transactions on Information Theory, vol. 51, no. 11, pp. 3820 - 3833, Nov. 2005.

[5] M. Uppal, V. Stankovic, and Z. Xiong, "Code designs for MIMO broadcast channels," in IEEE International Symposium on Information Theory, July 2006, pp. $2627-2631$.

[6] Y. Sun, M. Uppal, A. D. Liveris, S. Cheng, V. Stankovic, and Z. Xiong, "Nested turbo codes for the costa problem," IEEE Transactions on Communications, vol. 56, no. 3, pp. 388 -399, March 2008.

[7] G. Shilpa, A. Thangaraj, and S. Bhashyam, "Dirty paper coding using sign-bit shaping and LDPC codes," in IEEE International Symposium on Information Theory Proceedings (ISIT), June 2010, pp. 923 -927.

[8] C. B. Peel, B. M. Hochwald, and A. L. Swindlehurst, "A vector-perturbation technique for near-capacity multiantenna multiuser communication- Part I: channel inversion and regularization," IEEE Transactions on Communications, vol. 53, no. 1, pp. 195 - 202, Jan. 2005.

[9] B. M. Hochwald, C. B. Peel, and A. L. Swindlehurst, "A vectorperturbation technique for near-capacity multiantenna multiuser communication - Part II: perturbation," IEEE Transactions on Communications, vol. 53, no. 3, pp. 537 - 544, Mar. 2005.

[10] M. Costa, "Writing on dirty paper (corresp.)," Information Theory, IEEE Transactions on, vol. 29, no. 3, pp. 439 - 441, may 1983.

[11] G. Forney Jr., "Trellis shaping," IEEE Transactions on Information Theory, vol. 38, no. 2, pp. 281 -300, Mar. 1992. 